\title{
Double-Donor Energy Structure in Concentric Quantum Rings under Magnetic Field and Hydrostatic Pressure
}

\author{
M.R. Fulla ${ }^{a, b}$, J.H. Marín $^{b}$, D. Londoño ${ }^{a}$, C.A. Duque ${ }^{c}$ And M.E. Mora-Ramos ${ }^{c, d, e}$ \\ ${ }^{a}$ Institución Universitaria Pascual Bravo, AA 6564 Medellín, Colombia \\ ${ }^{b}$ Escuela de Física, Universidad Nacional de Colombia, AA 3840, Medellín, Colombia \\ ${ }^{c}$ Grupo de Materia Condensada-UdeA, Instituto de Física, Facultad de Ciencias Exactas y Naturales \\ Universidad de Antioquia UdeA, Calle 70 No. 52-21, Medellín, Colombia \\ ${ }^{d}$ Facultad de Ciencias, Universidad Autónoma del Estado de Morelos, CP 62209, México \\ ${ }^{e}$ Física Teórica y Aplicada, Escuela de Ingeniería de Antioquia, AA 7516, Medellín, Colombia
}

\begin{abstract}
A model for an artificial hydrogen molecule consisting of two positive on-axis Coulombic centers and two electrons coupled to them inside a double concentric quantum ring is considered. Such a nanostructure is assumed to be under the influence of external probes like hydrostatic pressure and magnetic field. By using the adiabatic approximation, the ground state energy is calculated as a function of the outer center line radius and the impurity Coulombic center separation, for different values of the hydrostatic pressure and magnetic field strength. In contrast to the single properties imposed by nature on the actual hydrogen molecule, our model allows us to explore a great variety of properties of the artificial hydrogen molecule by changing the ring dimensions. The artificial hydrogen molecule energy structure may be tuned by changing the external field strengths.
\end{abstract}

DOI: $10.12693 /$ APhysPolA.125.220

PACS: 71.55.Eq, 73.20.Hb, 73.21.La

\section{Introduction}

A wide variety of semiconductor nanostructures with different morphologies, confining electrons and holes in all three directions, have been grown in the last two decades. Among them, the ring-shaped one has attracted much attention because its singular - but nontrivial morphology offers us a unique system to analyze quantum interference effects [1], and to develop novel opto-electronic devices [2, 3]. Besides, it is well known that the presence of impurities in nanostructures modifies substantially the electro-optic and kinetic properties. However, there are few theoretical researches on the energy structure of hydrogenic impurities confined in quantum rings (QRs) [4-8]. Although there have been several reports on theoretical works on electrons and holes in double concentric quantum rings (DCQRs) [9], only very scarce studies on impurities in such systems have been already put forward.

Stimulated by these facts, we analyze here an on-axis two-hydrogenic molecule $\left(D_{2}^{0}\right)$ confined in DCQRs. We also assume that this system is under external probes like hydrostatic pressure and magnetic field. We address in detail the incidence of these fields on the $D_{2}^{0}$ salient features (equilibrium length and dissociation energy) as well as the effect of the donor position and the QRs geometrical parameters on the $D_{2}^{0}$ energy structure. This contribution is organized as follows. In Sect. 2, we obtain the basic formulae from the $D_{2}^{0}$ Hamiltonian by using adiabatic approximation. Section 3 presents the results and discussion. Finally, the remarkable conclusions are summarized in Sect. 4.

\section{Theoretical framework}

The $D_{2}^{0}$ molecular complex considered here is formed by two fixed and positive Coulombic centers at $\left(0,0, \xi_{1}\right)$ and $\left(0,0,-\xi_{2}\right)$, that share electrostatic coupling with two electrons with position vectors $\boldsymbol{r}_{1}$ and $\boldsymbol{r}_{2}$. These electrons are spatially separated and forced to move inside two concentric QRs with identical rectangular cross-sections whose dimensions are $W$ (QRs width) and $L$ (QRs height). A schematic plot of $D_{2}^{0}$ in DCQRs is shown in Fig. 1, where $R_{1}$ and $R_{2}$ are the inner and outer center line radii, respectively. The study takes into account the influence of the hydrostatic pressure by introducing the corresponding dependences of the electron effective mass and the dielectric constant on $P$ (measured in kbar): $m^{*}(P) / m_{0}=0.0665+5.7076 \times 10^{-4} P, \varepsilon(P)=$ $12.25-0.027 P$. The ring dimensions change according to the expression $L(P)=L(0)\left[1-\left(S_{11}+2 S_{12}\right)\right]^{1 / 2}$, whereas the radial size is $\rho_{j}(P)=\rho_{j}(0)\left[1-2\left(S_{11}+2 S_{12}\right)\right]^{1 / 2}$, and $\xi_{j}(P)=\xi_{j}(0)\left[1-\left(S_{11}+2 S_{12}\right)\right]^{1 / 2}$.

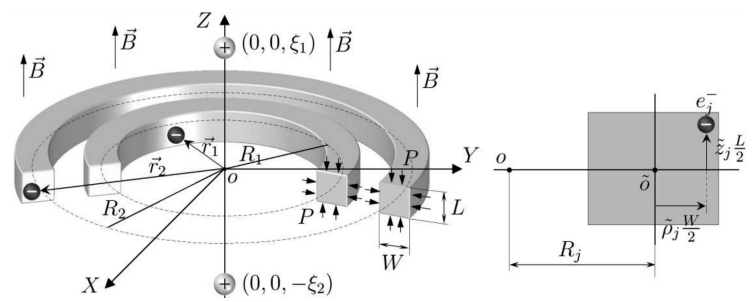

Fig. 1. Schematic 3D diagram of $C_{2}^{0}$ molecular complex in two concentric quantum rings under hydrostatic pressure and magnetic field.

In the effective-mass approximation, the $D_{2}^{0}$ Hamiltonian under the effects of hydrostatic pressure, and $z$-direction magnetic field, $\boldsymbol{B}$, can be written in cylindrical coordinates as follows: 


$$
\begin{aligned}
& H=\sum_{j=1}^{2} H_{0}\left(\boldsymbol{r}_{j}\right)+\frac{e^{2}}{\varepsilon(P)\left|\boldsymbol{r}_{2}-\boldsymbol{r}_{1}\right|}+\frac{e^{2}}{\varepsilon(P)\left|\boldsymbol{\xi}_{2}-\boldsymbol{\xi}_{1}\right|} \\
& H_{0}\left(\boldsymbol{r}_{j}\right)=-\frac{\hbar^{2}}{2 m^{*}(P)}\left[\frac{1}{\rho_{j}} \frac{\partial}{\partial \rho_{j}}\left(\rho_{j} \frac{\partial}{\partial \rho_{j}}\right)\right. \\
& \left.\quad+\frac{1}{\rho_{j}^{2}} \frac{\partial^{2}}{\partial \varphi_{j}^{2}}+\frac{\partial^{2}}{\partial z_{j}^{2}}\right]+\frac{\mathrm{i} e \hbar B}{2 m^{*}(P)} \frac{\partial}{\partial \varphi_{j}}+\frac{e^{2} B^{2} \rho_{j}^{2}}{8 m^{*}(P)} \\
& \quad+\sum_{k=1}^{2} \frac{e^{2}}{\varepsilon(P)\left|\boldsymbol{r}_{j}-\boldsymbol{\xi}_{k}\right|}+V\left(\rho_{j}, z_{j}\right) \\
& \left|\boldsymbol{r}_{2}\left(\rho_{2}, \varphi_{2}, z_{2}\right)-\boldsymbol{r}\left(\rho_{1}, \varphi_{1}, z_{1}\right)\right| \\
& \quad=\sqrt{\rho_{1}^{2}+\rho_{2}^{2}-2 \rho_{2} \rho_{1} \cos \left(\varphi_{2}-\varphi_{1}\right)+\left(z_{2}^{2}-z_{1}^{2}\right)} \\
& \left|\boldsymbol{r}_{j}\left(\rho_{j}, \varphi_{j}, z_{j}\right)-\boldsymbol{\xi}_{k}\right|=\sqrt{\rho_{j}^{2}+\left(\xi_{k}-z_{j}\right)^{2}} .
\end{aligned}
$$

The confinement potential $V\left(\rho_{j}, z_{j}\right)$ is assumed to be zero (infinite) inside (outside) the QR region. The Coulomb interaction terms appearing in Eq. (2.1) prevent from obtaining exact eigenvalues. In consequence, an approximated method must be used in order to solve the corresponding Schrödinger equation. For this, we can take into account the structural properties of self-assembled DCQRs [10]. In accordance, we notice that the height-to-center line radius aspect ratio is very small $\left(L / R_{j} \ll\right.$ 1 , for $j=1,2)$. Taking into account this fact and considering, for the sake of mathematical convenience, the situation of very narrow $\mathrm{QRs}\left(W / R_{\mathrm{c}} \ll 1\right)$, we may use a numerical procedure based on the adiabatic approximation (AA) $[4,11]$. Firstly, we carry out a rescaling of the coordinates: $\rho_{j}=\tilde{\rho}_{j} W / 2+R_{j}$ and $z_{j}=\tilde{z}_{j} L / 2$, where the new dimensionless variables $\tilde{\rho}$ and $\tilde{z}$ take values inside the $\mathrm{QR}$ between -1 to 1 . The use of the AA scheme, which is computationally faster than the diagonalization and variational methods, allows us to decouple the slow electron motion along the $z$-axis from the fast transverse electron motion within the QRs cross-section. Accordingly, the equation with a three-dimensional Hamiltonian reduces to the following one-dimensional eigenvalue problem:

$$
\begin{aligned}
H & =\sum_{j=1}^{2}\left[-\frac{\hbar^{2}}{2 m^{*}(P) R_{j}^{2}} \frac{\partial^{2}}{\partial \varphi_{j}^{2}}+\frac{\mathrm{i} e \hbar B}{2 m^{*}(P)} \frac{\partial}{\partial \varphi j}\right. \\
& \left.+\frac{e^{2} B^{2} A_{j}\left(\varphi_{j}\right)}{8 m^{*}(P)}\right]+\bar{V}_{\mathrm{ee}}\left(\varphi_{2}-\varphi_{1}\right) \\
& +\sum_{j=1}^{2} \bar{V}_{j}+\frac{\eta e^{2}}{\varepsilon_{2}(P)\left|\boldsymbol{\xi}_{2}-\boldsymbol{\xi}_{1}\right|}+E_{0},
\end{aligned}
$$

where

$$
\begin{aligned}
& A_{j}(\varphi)=\int_{0}^{1} \tilde{g}_{j}^{2}\left(\tilde{\rho}_{j}, \varphi\right)\left(R_{j}+\tilde{\rho}_{j} W / 2\right)^{2} \tilde{\rho}_{j} \mathrm{~d} \tilde{\rho}_{j}, \\
& \bar{V}_{\text {ee }}\left(\varphi_{2}-\varphi_{1}\right)=\frac{e^{2}}{\varepsilon(P)} \int_{-1}^{1} \int_{-1}^{1} \int_{-1}^{1} \int_{-1}^{1}
\end{aligned}
$$

$$
\begin{aligned}
& \frac{f_{1}^{2}\left(\tilde{\rho}_{1}, \varphi_{1}\right) f_{2}^{2}\left(\tilde{\rho}_{2}, \varphi_{2}\right) g_{1}^{2}\left(\tilde{z}_{1}, \varphi_{1}\right) g_{2}^{2}\left(\tilde{z}_{2}, \varphi_{2}\right)}{\left|\boldsymbol{r}_{2}\left(\tilde{\rho}_{2}, \varphi_{2}, \tilde{z}_{2}\right)-\boldsymbol{r}\left(\tilde{\rho}_{1}, \varphi_{1}, \tilde{z}_{1}\right)\right|} \\
& \times \tilde{\rho}_{1} \tilde{\rho}_{2} \mathrm{~d} \tilde{\rho}_{1} \mathrm{~d} \tilde{\rho}_{2} \mathrm{~d} \tilde{z}_{1} \tilde{z}_{2}, \\
\bar{V}_{j} & =\frac{-e^{2}}{\varepsilon(P)}\left[\int_{-1}^{1} \int_{-1}^{1} \frac{f_{j}^{2}\left(\tilde{\rho}_{j}, \varphi_{j}\right) g_{j}^{2}\left(\tilde{z}_{j}, \varphi_{j}\right)}{\left|\boldsymbol{r}_{j}\left(\tilde{\rho}_{j}, \varphi_{j}, \tilde{z}_{j}\right)-\boldsymbol{\xi}_{1}\right|} \tilde{\rho}_{j} \mathrm{~d} \tilde{\rho}_{j} \mathrm{~d} \tilde{z}_{j}\right. \\
& \left.+\int_{-1}^{1} \int_{-1}^{1} \frac{f_{j}^{2}\left(\tilde{\rho}_{j}, \varphi_{j}\right) g_{j}^{2}\left(\tilde{z}_{j}, \varphi_{j}\right)}{\left|\boldsymbol{r}_{j}\left(\tilde{\rho}_{j}, \varphi_{j}, \tilde{z}_{j}\right)-\boldsymbol{\xi}_{2}\right|} \tilde{\rho}_{j} \mathrm{~d} \tilde{\rho}_{j} \mathrm{~d} \tilde{z}_{j}\right] .
\end{aligned}
$$

Here, $f_{j}(\tilde{\rho}, \varphi) g_{j}(\tilde{z}, \varphi)$ is the normalized ground state electron wave function in an infinite two-dimensional square quantum well whose lowest energy value is $E_{0}\left(\tilde{\rho}_{j}, \tilde{z}_{j}\right)=$ $\left[\pi^{2} \hbar^{2} / 2 m^{*}(P)\right]\left(1 / L^{2}+1 / W^{2}\right)$. The dynamics of the two electrons may be easily described by using center of mass, $\Theta=\frac{R_{1}^{2} \varphi_{1}+R_{2}^{2} \varphi_{2}}{R_{1}^{2}+R_{2}^{2}}$, and relative angular, $\varphi=$ $\varphi_{2}-\varphi_{1}$, coordinates. In these coordinates, the Hamiltonian (2.2) can be rewritten as $H=H_{\Theta}+H_{\Phi}$, where the center-of-mass and relative terms, respectively, satisfy the equations: $H_{\Theta} \Psi_{M}(\Theta)=E_{\Theta}(M) \Psi_{M}(\Theta)$ and $H_{\Phi} \Phi_{m, s}(\varphi)=E_{\Phi}(m, s) \Phi_{m, s}(\varphi)$. The Schrödinger equation with Hamiltonian $H_{\Theta}$ can be solved exactly, whilst the other one has to solve numerically with periodic conditions in the region $[-2 \pi, 2 \pi]$ defined by the expression $\Phi_{m, s}(0)=(-1)^{m} \Phi_{m, s}(2 \pi)$. The quantum numbers $M=0, \pm 1, \pm 2, \ldots$, and $m=0, \pm 1, \pm 2, \ldots$, define the center-of-mass angular momentum and the two-electron relative angular momentum, respectively. On the other hand, $s=+$ denotes even solutions or singlet states, whilst $s=-$ denotes the odd solutions or triplet states.

\section{Results and discussion}

In Fig. 2 we show the renormalized effective potential $\bar{V}=\bar{V}(\varphi)$ as a function of the relative coordinate $\varphi$ for three values of the hydrostatic pressure: $0,15 \mathrm{kbar}$, and $30 \mathrm{kbar}$, without applied magnetic field. This potential governs the rotational effects for a hypothetical particle with reduced mass in the Schrödinger equation corresponding to the relative term. We can see that the greater is the hydrostatic pressure applied, the deeper is the effective potential. This fact is a result of the reduction in the overall size of the system, the increase of the electron effective mass, and the fall in the dielectric constant. All this leads to the reinforcement of the electron-impurity coupling.

The $D_{2}^{0}$ ground state energy as a function of the outer center line radius is shown in Fig. 3 for three different values of the hydrostatic pressure. We notice from this figure that the total energy tends to augment with the increasing outer center line radius. This is due to the reduction of the strength of both the attractive interaction between the two-Coulombic centers and the second electron into the outer QR and the electron-electron repulsive interaction. Nevertheless, the above mentioned behavior tends to change for very large outer center line radius $\left(R_{2}>400 \mathrm{~nm}\right)$ because the slopes for all three curves tend to be zero. In these cases, the second QR is far enough from the first ring and the impurities. For 


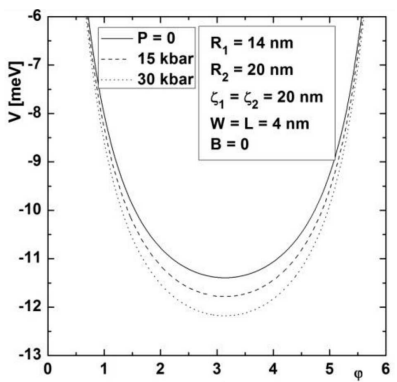

Fig. 2. Renormalized effective potential of $D_{2}^{0}$ in a GaAs double concentric quantum ring as a function of the relative angular coordinate $\varphi$.

this reason, the $D_{2}^{0}$ energy tends asymptotically to the $D_{2}^{+}$ground state energy. In consequence, by increasing the outer center line radius we are able to analyze the dissociation process: $D_{2}^{0} \rightarrow D_{2}^{+}+\mathrm{e}^{-}$. We can also notice that the effect of a nonzero applied pressure is to lower the ground state energy.

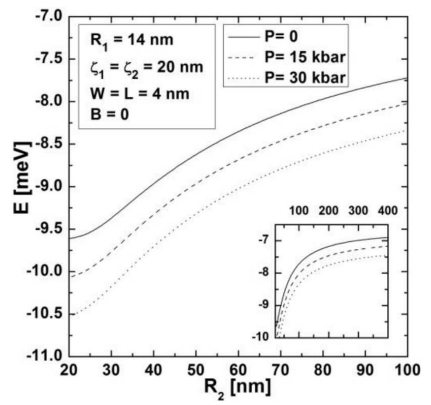

Fig. 3. $D_{2}^{0}$ ground state energy as a function of the outer center line radius for three different values of the hydrostatic pressure: 0,15 , and $30 \mathrm{kbar}$. The inset shows the curve behaviors for larger values of the outer center line radius.

In Fig. 4, we display the results of $D_{2}^{0}$ calculation of ground state energy as a function of the separation between the Coulombic centers, for different values of the hydrostatic pressure $(P=0,15 \mathrm{kbar}$, and $30 \mathrm{kbar}$ - part (a)) and different values of the magnetic field $(B=0.2 \mathrm{~T}$, and $3.4 \mathrm{~T}-$ part $(\mathrm{b}))$. These curves present an actual $\mathrm{H}_{2}$-like behavior, because the all have deep minima for impurity separation distance equal to $20 \mathrm{~nm}$. Indeed, the salient features of the actual $\mathrm{H}_{2}$ complex are predetermined by the nature, but the salient features of $D_{2}^{0}$ may be changed by modifying the external strength fields applied on the DCQR as well as the QR's geometrical parameters. On the one hand, the increase of the hydrostatic pressure yields values of the dissociation energy much greater in comparison with those of the DCQRs at zero pressure. In this regard, the increase of the hydrostatic pressure allows us to obtain artificial molecules much stable against the dissociation. The inset in the left part shows that all curves tend to merge as long as the impurity-impurity separation is large enough. On the other hand, the increase of the magnetic field strength shifts up energy minima, which tends to destroy the $D_{2}^{0}$ molecular complex stability. This happens because the dissociation energy exhibits a decrease when the magnetic field is turned on. This effect is due to the fact that a nonzero magnetic field pushes the electrons toward the internal QR's wall, forcing them to move faster around the $z$-axis. Then, there is an increase of the electronic kinetic energy which leads to destroying the molecular identity due to the great competition between the attractive potential energy and the positive electronic kinetic energy. Finally, these results illustrate a wide range of possibilities to tune in real time the $D_{2}^{0}$ dissociation energy or the stability of this molecular complex by changing the external probes.

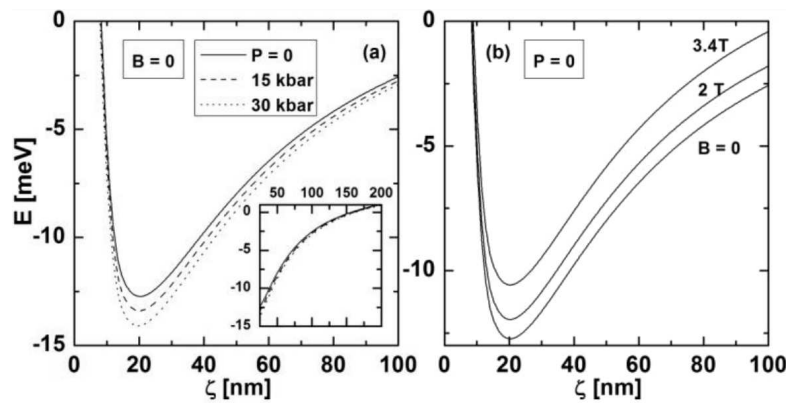

Fig. 4. $\quad D_{2}^{0}$ total energy as a function of the impurityimpurity separation for three different values of the hydrostatic pressure: $0,15 \mathrm{kbar}$, and $30 \mathrm{kbar}$ (a) and for three different values of the magnetic field strength 0 , $2 \mathrm{~T}$, and $3.4 \mathrm{~T}(\mathrm{~b})$.

The evolution of $D_{2}^{0}$ total energy with the magnetic field strength is shown in Fig. 5. The states are associated with different values of the quantum numbers $M$, $m$, and $s$.

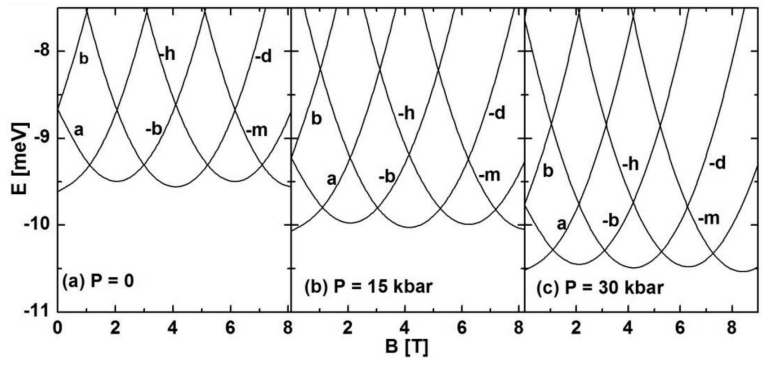

Fig. 5. $\quad D_{2}^{0}$ total energy as a function of magnetic field strength for three values of the hydrostatic pressure: 0 , 15 kbar, and 30 kbar.

We have: $a \rightarrow(0,0,0), b \rightarrow(1,1,1),-b \rightarrow(-1,1,1)$, $-d \rightarrow(-2,0,0),-h \rightarrow(-3,1,1),-m \rightarrow(-4,0,0)$. The noticeable evolution of these curves is the result of the strong competition between the paramagnetic term (term varies linearly with the magnetic field) and the 
diamagnetic one (term proportional to the square of the magnetic field). For instance, when $M \geq 0$ all curves are linear at the beginning with positive slope. When the quantum number $M$ is negative, the slope of the curve becomes negative for small values of the magnetic field strength. But, then, the parabolic term in $B$ dominates the competition making the energy to begin a growth with the magnetic field. The result is the appearance of periodic oscillations of the ground state energy known as the Aharonov-Bohm oscillations. From these figures we can see that the increase of the hydrostatic pressure yields a little increase in the oscillation period. This happens because the period is strongly dependent on the quantum ring size and the hydrostatic pressure modifies the quantum rings dimensions.

Additionally, the hydrostatic pressure shifts down the ground state energy level. For instance, a pressure $P=15$ kbar lowers the ground state in comparison with the zero pressure case in $\approx 4 \%$, whilst the pressure $P=30$ kbar lowers the ground state energy in $\approx 8 \%$. This fact seems to suggest that the $D_{2}^{0}$ ground state energy decreases linearly with the pressure applied on the DCQRs, which could not be surprising because a similar behavior was previously observed in quantum wells $[6,12]$. In order to give an answer to this suggestion, we plot the ground state energy in Fig. 5 as a function of the hydrostatic pressure.

The corresponding results are shown in Fig. 6. In this graphics there is shown that $D_{2}^{0}$ state energy displayed in Fig. 5 decreases linearly with the hydrostatic pressure applied on the DCQR according to the equation: $E_{\min -A B}=-0.030 P-9.6136$.

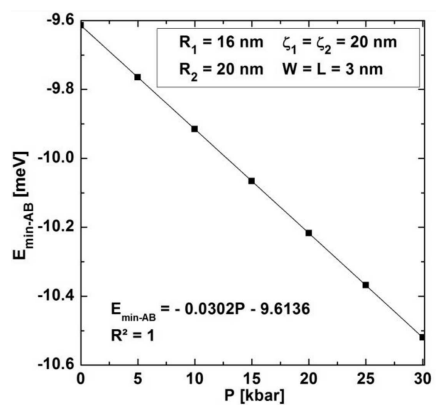

Fig. 6. $\quad D_{2}^{0}$ ground state energy displayed in Fig. 5 as a function of the hydrostatic pressure.

\section{Conclusions}

The salient features of an artificial molecule confined in a double concentric quantum rings have been studied by using a simple model based on the adiabatic approximation. The equilibrium length and the dissociation energy are strongly dependent on the external fields applied as well as on the double concentric quantum ring geometrical parameters. The application of a magnetic field tends to diminish the molecular stability while the hydrostatic pressure tends to favor the molecular stability. Finally, an artificial hydrogen molecule can be obtained by changing the geometrical parameters and its energy spectrum may be tuned in real time by changing the external field strengths. This fact allows us to modify the $D_{2}^{0}$ essential properties in an easy way, and obtain a wide variety of artificial molecules whose properties are actually different from the real hydrogen molecule $\mathrm{H}_{2}$.

\section{Acknowledgments}

M.E.M.R. acknowledges support from Mexican CONACYT through grant CB-2008-101777, and through sabbatical grant 2011-2012 No. 18036. He also thanks Universidad de Antioquia and Escuela de Ingeniería de Antioquia for hospitality during his sabbatical stay. This research was partially supported by Colombian Agencies: CODI-Universidad de Antioquia (Estrategia de Sostenibilidad 2013-2014 de la Universidad de Antioquia and the project: E01535-Efectos de la presión hidrostática y de los campos eléctrico y magnético sobre las propiedades ópticas no lineales de puntos, hilos y anillos cuánticos de GaAs-(Ga,Al)As y $\left.\mathrm{Si} / \mathrm{SiO}_{2}\right)$, Facultad de Ciencias Exactas y Naturales-Universidad de Antioquia (CAD-exclusive dedication project 2012-2013), and by El Patrimonio Autónomo Fondo Nacional de Financiamiento para la Ciencia, la Tecnología y la Innovacion, Francisco José de Caldas. The work was developed with the help of CENAPAD-SP, Brazil.

\section{References}

[1] G. Timp, A. Chang, J. Cunningham, T. Chang, P. Mankiewich, R. Behringer, R. Howard, Phys. Rev. Lett. 58, 2814 (1987).

[2] R. Vrijen, E. Yablonovitch, K. Wang, H.W. Jiang, A. Balandin, V. Roychowdhury, T. Mor, D. DiVincenzo, Phys. Rev. A 62, 012306 (2000).

[3] D. Loss, D.P. DiVincenzo, Phys. Rev. A 57, 120 (1998).

[4] F.J. Betancur, W. Gutiérrez, J.C. Piña, Physica B 396, 12 (2007).

[5] S. Kang, Y.M. Liu, T.Y. Shi, Commun. Theor. Phys. 50, 767 (2008).

[6] H. Akbas, I. Erdogan, O. Akankan, Superlatt. Microstruct. 50, 80 (2011).

[7] M.G. Barseghyan, M.E. Mora-Ramos, C.A. Duque, Eur. Phys. J. B 84, 265 (2011).

[8] A. Bruno-Alfonso, A. Latgé, Phys. Rev. B 61, 15887 (2000).

[9] F.J. Culchac, N. Porras-Montenegro, A. Latgé, J. Appl. Phys. 105, 094324 (2009).

[10] T. Mano, T. Kuroda, S. Sanguinetti, T. Ochiai, T. Tateno, J. Kim, T. Noda, M. Kawabe, K. Sakoda, G. Kido, N. Koguchi, Nano Lett. 5, 425 (2005).

[11] J.H. Marín, M.R. Fulla, F. Rodríguez-Prada, F. García, J.C. Piña, Superlatt. Microstruct. 49, 258 (2011).

[12] A.L. Morales, A. Montes, S.Y. López, C.A. Duque, J. Phys., Condens. Matter 14, 987 (2002). 